\title{
Is telepathology worth being developed?
}

\author{
Włodzimierz Olszewski
}

In pathology, similarly to other branches of medicine, and in particular in the field of visualisation techniques, the use of image transmission via the Internet for diagnostic or consulting purposes is being introduced. One of the reasons for this is the limited number of specialists. Currently, three basic applications of this technique can be distinguished: telediagnostics, teleconsultations and teleconferences.

In contrast to teleradiology where the basic subject of analysis is the image obtained by various visualisation techniques - in pathology, the basis is tissue or cell analysis. The evaluation of such material should be from the start in the hands of pathomorphologists. The transmitted image of the performed histological preparation may not be fully representative of the lesion being examined (cancer). In modern oncological pathomorphology, it is very often necessary to use special techniques such as immunohistology or molecular biology techniques to determine the proper diagnosis. Performing such tests requires direct access to tissue or cytological material.

Telepathology can be used as part of consultations between reference centres. Modern telepathology techniques allow the use of these methods for training purposes, including the interactive participation of trainees.

NOWOTWORY J Oncol 2017; 67, 6: 372-374

Key words: telepathology, frozen section, consultation, pathology education

Within the annual oncological debates organised by the Editor's Office of the Nowotwory Journal of Oncology, I was invited to participate in a discussion concerning the status of telepathology, or to be more precise, to present my opinion whether this field of pathomorphology should be developed and, if so, introduced to practice. My standpoint presented in these contributions - in the opinion of the organisers of the debate - should be negative.

Essentially, I decided to present my opinion on the place of telepathology in contemporary pathomorphological diagnostics, paying special attention to its limitations and to the benefits in the application of this technique.

An interest in the use of telepathology was the outcome, among others, of the limited number of pathomorphologists and the lack of possibility of performing pathological assessment, also those of a perioperative nature, in local hospitals.

The first reports regarding the possibility of using this technique (method) of preparing and evaluating sections concerned the situation in which it was not possible to evaluate the sections in a given hospital so it was necessary to send microscopic images to a pathologist in another hospital.

It was therefore assumed that it would be sufficient if a trained hospital staff member e.g. a medical technician, could collect a tissue section and make a histological specimen, and then the microscopic image could be sent to a pathomorphologist in a centre any distance from the place of the surgery. A pathomorphologist, on the basis of the image rendered on the computer screen, would establish a diagnosis and then communicate the result on the telephone allowing for further therapeutic steps.

The development of visualisation and endoscopic techniques resulted in a decrease of the need to perform a peri-operative assessment with the purpose of establishing whether a cancer is malignant or not and what further stages of the surgery should be. Currently, one of the most frequent indications for peri-operative examination is the assessment of surgical margins and such surgeries are performed in centres which have the appropriate pathomorphological facilities.

Currently, three basic applications of telepathology may be distinguished: 
— tele-diagnostics

- tele-consultations,

- teleconferences [1].

Tele-diagnostics, as was mentioned in the Introduction here, was the initial purpose of using telepathology with regards to the limited number of specialists (pathomorphologists) for perioperative assessment.

It must be remembered, however, that the basic and initial part of an examination is the macroscopic evaluation of the specimen obtained for examination and a detailed selection of the tissue fragment from which the specimen for a peri-operative evaluation will be made. This action concerns both perioperative assessment and a detailed post-operative examination and forms the only significant imitation of the value of an examination made remotely for a macroscopic evaluation.

In telemedicine, the largest development is observed (found) in tele-radiology, both with respect to traditional diagnostics, and to more recent visualisation techniques (ultrasound, CT, PET-MR). The basic difference between tele-radiology and telepathology consists in the fact that in teleradiology, the very image, which can be transmitted remotely, is important, whilst in pathomorphology, the core element is the tissue (cell) which is the basis of the diagnostic workout. In pathology, this is defined as: the tissue or cell is the issue - what matters is the tissue or the cell and not the image alone.

In contemporary oncology, especially in the case of some of the most common cancers, such as breast or lung cancer, the core element of pathomorphological diagnosis, gaining an increasing interest, is the evaluation of predictive factors with the use of immunohistochemistry or molecular biology methods. Such examination requires an access to the tissue material, usually formalin-fixed paraffin blocks or cytology specimens. This diagnostic procedure is also required in many rare cancers such as soft tissue sarcomas. In such cases, the tissue or cytology specimens are sent to reference centres which specialise in the diagnostics of a group of rare cancers. The necessity of such a way of management in pathomorphological diagnostics poses the most significant limitation for the extensive application of telepathology.

Initially, a static, selected microscopic image was transmitted. Currently, a scanned image of the entire specimen can be sent. This procedure is, however, very time-consuming. The recently introduced technological solutions allow for a remote control of the microscope during the pathomorphological assessment. This type of telepathology is defined as dual-modality systems (digital pathology systems) that combine whole slide imaging (WSI) and real-time dynamic robotic telepathology [2].

A significant limitation of telepathology is also the fact that although this method can be and should be applied in some selected exceptional cases, it must be remembered that here a pathologist is unable to analyse a few images on a few screens at the same time. Pathomorphology is still a fairly subjective method, and the number of assessments performed by a pathologist in a particular time period is limited. Another important aspect is the significance of pathomorphological diagnosis in oncology, as this diagnosis plays a decisive role in therapeutic decisions. A pathomorphologist evaluating a histological or a cytological image and establishing a diagnosis should, in their interpretation, consider the entire clinical picture, for example the data concerning previous treatment. Telepathology may only partly solve the problem in an insufficient number of pathomorphologists.

There have been numerous attempts to replace screening of cytological specimens made by cyto-morphologists by a programmed system of automated selection of specimens for further diagnostics. None of the proposed programs have been finally accepted in medical practice yet. The main reason was the lack of possibility of false negative results.

Telepathology is currently applied in consultations, where the microscopic image is transferred, frequently together with the image of the performed immunohistochemistry examination, to the reference centre which deals with the diagnostics of rare cancers. Some difficulty may lie in the diversity of modern techniques of registration and transmission of the microscopic image, and resulting incompatibility of the equipment. This problem, however, is undoubtedly to be solved.

It is certain that telepathology can be applied at tele-conferences when carefully prepared material (e.g. additional staining) is sent for consultation in order to confirm diagnosis. It is possible to have the image evaluated simultaneously by a few pathologists who may exchange opinions, which not only allows for establishing a correct diagnosis, but also provides possibilities of improving professional qualifications and sharing experience.

The application of telepathology for training purposes is increasing in popularity. It provides for a possibility of evaluating the previously scanned entire histopathological or cytological specimens, allowing for a complete assessment of a specimen, in a method similar to the conventional one. In many universities, the students, during their pathomorphology class, instead of using microscopes, analyse correctly scanned histopathological or cytological specimens, having also a possibility of adjusting the magnification.

It is worthwhile, however, that the majority of experienced pathomorphologists are more inclined to participate in conferences at which it is possible to evaluate specimens in a microscope and not on an LCD screen. This can be compared to the preference of reading paper books over the use of e-books. It is quite likely, however, that a new generation 
of pathomorphologists who are currently being educated at medical universities, where they already use the screen-rendered images in class instead of a microscope, will have a different attitude to the evaluation of pathomorphological images presented on computer screens.

\section{Conclusion}

To sum up, one may say that telepathology is and should be applied for training purposes (courses, conferences) and sometimes, it allows for consulting selected cases [3]. The necessity to have a tissue or cytology specimen does not allow for replacing the basic, conventional pathomorphological diagnostics with telepathology methods. Moreover, the introduction of biological methods for routine diagnostic management would require a close collaboration between the pathomorphologist and molecular biologist.

It may be concluded beyond any doubt that telepathology will not be able to solve the issue of the lack of pathomorphologists.

\section{Conflict of interest: none declared}

\section{Prof. Włodzimierz Olszewski, MD, PhD}

Maria Skłodowska-Curie Memorial Cancer Center

and Institute of Oncology

Department of Pathology

ul. Roentgena 5, 02-781 Warszawa, Poland

e-mail:wtolszewski@coi.pl

Received \& Accepted: 15 Jan 2018

Based on the presentation at the V Annual Conference of the Nowotwory Journal of Oncology, 'Oncological Debates', held in Warszawa, 7-8th April 2017

\section{References}

1. Thrall M. Pantanowitz L. Khulbuss W. Telecytology: Clinical applications, current challenges, and future benefits. J Pathol Inform 2011; 2:51.

2. Braunhut BL, Graham AR, Richter LC et al. Fifth generation of telepathology system. Workflow analysis of the robotic dynamic telepathology component. Diagnostic Pathol 2013; 8 (Suppl 1): S3.

3. Van Es SL, Kumar RK, Pryor WM et al. Cytopathology whole slide images and adaptive tutorials for postgraduate pathology trainees: a randomized crossover trial. Hum Pathol 2015; 46: 1297-1305. 